\title{
Decreased Peri-Insular Halos
}

National Cancer Institute

\section{Source}

National Cancer Institute. Decreased Peri-Insular Halos. NCI Thesaurus. Code C158334.

Decreased number and/or size of peri-insular halos. 